\title{
Re-thinking digital design
}

\author{
R. Oxman \\ Faculty of Architecture and Town Planning, Technion, Haifa, Israel
}

\begin{abstract}
Designerly ways of thinking have become a significant topic in design research. If indeed, the contemporary phenomena of "digital design thinking" are different from traditional models, then there is emerging pressure to pioneer new teaching paradigms Theories and methods of digital design can no longer be conceptualized as the merging of computational tools with conventional formulations of design thinking. Within the framework of this orientation to a critical formulation of new educational agenda, pedagogical issues are considered. A new orientation to understanding the impact of digital media on "digital design thinking" and pedagogy is presented, discussed and demonstrated.
\end{abstract}

Keywords: digital design, digital architecture, digital design thinking.

\section{Introduction}

Theories and methods of digital design can no longer be conceptualized as the merging of computational tools with conventional formulations of design. There is the need to pioneer a new understanding of the nature of designing in relation to digital design media. If the very nature of design is radically changing, how then can we accommodate and recognize emerging theories of design as the basis for a new pedagogy? It has now become important to consider the significance of terms such as "digital design thinking" (Oxman [15]) and what they might imply with respect to new approaches of design education. If digital design thinking constitutes a new conceptualization, including concepts as the meaning of form, the nature of functional and formal knowledge in design, and generative processes, then there is a need for a new pedagogy.

The conventional educational model in the design studio generally employs a simulation of praxis as a didactic model. That is, the didactic stages are driven by a theoretical interpretation of program, site and conditions carried through stages 
of conceptualization, schematic design and design development. Furthermore, most studios still employ well accepted knowledge-bases and typologies as well as traditional paper-based sketches as media of what was referred to as a conceptual and explorative medium. Schön's classic characterization of visual reasoning in the design as a "dialogue with the materials of the problem" and the process of "backtalk" from visual images (Schön [17]) are still the dominant model for teaching in the design studio. However, as we attempt to re-evaluate the logic of the Schön model, we find the need to re-define the concept of "material" and to understand the impact of digital design in design thinking.

Designerly ways of thinking have become a significant topic in design research (Lawson [8]). However, if indeed, contemporary phenomena of "digital design thinking" are different from traditional models, than there is emerging pressure to pioneer new teaching paradigms (Kvan et al. [6]). Within the framework of this orientation to a critical formulation of new educational agenda, the following issues are considered:

- Are we encountering new paradigms of design, or are we essentially encountering the same cognitive phenomena of known processes of design thinking in the new digital media?

- Is digital design so different from traditional paper-based design that many of our root concepts must be reformulated? If this is the case, how then, can we begin to conceptualize and formulate "digital design thinking"?

- Furthermore, if conventional teaching approaches are obsolete, what are we teaching when we teach about the design media? Are we, in fact, teaching novel design paradigms?

In the context of an experimental design studio we explore and identify these issues, evaluate findings, suggest and test appropriate new didactic principles. The objectives of our initial experimental studio are to take first steps through a process rethinking many of the root assumptions of current computational conventions. We determine the relevance of these findings for conceptualizing new pedagogy in the design studio, and carried out and evaluated these approaches in a series of experimental studios.

\section{Towards a new rationale}

The evolution of digital design as a unique field of design endeavor, motivated by its own body of theoretical sources, and a culture of discourse, is beginning to evolve unique ideology, methodologies and formal content (Oxman [15]; Liu [9]; Kolarevic [5]). Given the growing amplitude of issues and subjects in digital design as witnessed by practice, research and education, we need to formulate a theoretical framework that is suitable to the formation of design educational theory. 


\subsection{The emergence of a new ideology}

Digital design thinking is more than simply a set of formal preferences. It is the abandonment of the modernist design ontology that is predicated upon formal and typological knowledge (e.g. formal languages, typological classes and generic design, etc.) It is non-typological and non-deterministic in supporting and preferring the differentiated over the discrete and the typological. There is emerging a new symbiosis between the digital product of design and the way it is conceived, generated and produced in digital media. These stages are fundamentally different from those of modernist design. It is the understanding and formulation of this procedural symbiotic relationship between conception, generation, production and the product itself that appears to be of high priority today. Digital technologies appear to have freed the image from traditional concepts of representation. We no longer represent discrete shapes in the conventional paper-based sense. This condition has enhanced the denial of classical notions of representational conventions such as static space, and has introduced new concepts of dynamic and responsive space and form that are producing new classes of designs.

In many cases approaches to form generation exploit emergence-based transformational processes in which digital media are the enabling environment. This in many ways is replacing the experimental visual nature of the paperbased sketching process. Context in the modernist sense may possess iconic, stylistic, or configurative content that can implicate design through visual or formal content. Context in digital design is considered a performative shaping force acting upon shape and form.

\subsection{Paradigmatic classes of digital design models}

These indications of conceptual change have emerged the formulation of design models, the conceptual content and vocabulary of digital design. A formulation through the identification of relevant early models of design has been developed by the author (Oxman [15]). The classification of paradigmatic models includes: CAD models, formation models, generative models, performance models and integrated compound models. This classification enables the definition of underlying current digital design models.

\subsubsection{CAD}

Early CAD models marked an attempt to depart from paper-based media. They had little qualitative effect on design in comparison to conventional paper-based models. In traditional CAD the interaction with formal representations supports the a posteriori automation of design drawings and visual models. First CAD systems were mainly descriptive, employing various geometrical modeling / rendering software.

\subsubsection{Formation}

In digital design the centrality of traditional concepts of paper-based representation are no longer valid conceptions for explicating the thinking and 
processes associated with digital design. Furthermore, in certain formation processes of digital design the formal implications of the concept of representation are negative and unproductive. Emerging design theory has transformed the concept of form into the concept of formation associated with topological, parametric and animation. Topological design is based on the exploitation of topology and non-Euclidean geometry. Parametric design is based on principles of parametric design (Burry [1]). And generative components, Animation, morphing (Lynn [12]) and other range of motion and time-based modeling techniques are based on the propagation of multiple discrete instantiations in a dynamic continuum.

\subsubsection{Generation}

Generative models of digital design are characterized by the provision of computational mechanisms for formalized generation processes. Here, as compared to formation models, shapes and forms are considered to be a result of pre-formulated generative processes. Currently there is a rich theoretical body of research-related applications of generative models. Two main distinct current sub-approaches are shape grammars (Stiny [18]; Knight and Stiny [7]) and evolutionary models (Frazer [4]).

\subsubsection{Performance}

Performance-based models are driven by performance and potentially integrated with formation and generative processes. Forces in a given context are fundamental to form-making in digital design. External forces may be considered as environmental forces including structural loads, acoustics, transportation, site, program etc. Information itself is also considered as an external "force" that can manipulate the design.

\section{The conceptual content of digital design}

We have attempted to build educational content by explicating the new conceptual structure of digital design. In reality, the integration and interaction of technological content with that of conceptual content is obviously part of the formative process of learning to design with media. However, the exploitation and experimentation with new concepts can prove to be an articulate environment for design learning (Oxman [19]) in which learning by making is transfigured by its conceptual, rather than computational, content. Given that a rigorous formulation of such emerging concepts does not yet exist, any work based upon an as yet unformulated body of theory must by necessity be in itself experimental.

\subsection{Beyond formal representational design}

The first stage of such a conceptual mapping is predicated upon the prevailing models of design at the level of their own conceptual structures. The prevailing model of modernist design is a formalist model in the profound sense of what we might term design ontology. Modernist design is formulated about the sequential 
development of symbolic representations of the design. It traditionally begins with considerations of space, with the major emphasis being upon the manipulation of visualizations of the design object -the design of form - through the stages of conceptual design, schematics, design development and materialization. The formal foundations of modern art and design have been theoretically defined and the evolutionary process of formal-graphical evolution in design representation has been well-formulated by various theoreticians.

We are now moving beyond this formal syndrome. The parametric, topological, geometric and generative characteristics of current digital design (Lynn [12]) are in profound theoretical contradiction to shape production in the formalist models. Irrespective of how unique that shape may be, it is still the process of shape production as the production of a static form. Digital design characterized by generative processes related to movement and time is neither formalistic nor static. Form generation, beyond formalism, produces conditions of pliancy and continuity in both the conception and geometry of form.

\subsection{Formation, generation and performance: implications of the models}

Formation, generation and performance are the motivating forces in the new design. They, as concepts and processes, begin to condition new design procedures that are uniquely conceptual. To some extent, these conceptual stages - in the establishment of an appropriate morphology for the design- are also noncontextual. Shreds, Strands, Bleps, Flowers and Folds are among Lynn's [12] interpretations of the morphologies of digital form.

First material, then generative procedure, and then performance appear to be the methodological sequence of digital design. It is this methodological sequence of procedures that supports the preference for time-related transformational states in place of the representation of static design representations.

This characterization of the digital design model is completely contradictory to models of design such as Schön's "reflective practitioner" in which the visual representation of the design is manipulated by visual reasoning through a succession of stages generally in the medium of sketching. This interpretation of sketching as design thinking through iterative stages of visual discovery is the antithesis of the digital model. Digital design brings new design ontology beyond the visual interpretation of form.

\subsection{Digital systems as the medium for design process}

The term digital design system, according to our definition implies the digital integration of attributes related to the morphology + structure + behavior of certain morphological-geometric classes of material form. Furthermore, in the studio the need for the integration of both the digital model and the physical model were found to be extremely meaningful for the conceptualization of digital material. Since current descriptive geometrical modeling lacks material and structural logic, the physical model provides a complementary medium. The physical model is still very useful for feel and touch in exploring principles of form, morphology and structure. Physical studies can then be translated into digital models for transformation and versioning. 


\section{Re-thinking digital design}

In the following section a didactic approach in guiding three different paradigmatic projects is presented and illustrated. Each project was developed by exploiting digital concepts and techniques that suited the theoretical and conceptual content of the project. Each conceptual basis presented the designer with a medium for the development of the material concept through its parametric and morphological evolution. In each of the following selected projects a conceptualization of digital material and a unique digital process appropriate to the material concept and to the type of media is presented. Our didactic process consists of the following four basic tasks: the first task is to conceptualize and test a generic type of digital material. The second task is to define a unique responsive strategy for modification. The third task is to select a generative model. The fourth task is to select a context that can best demonstrate the behavior and applicability of the design material in relation to task specifications. In the following sections we demonstrate and illustrate these didactic steps in a series of selected projects.

\subsection{Topological design: "The Boundary Wall"}

The first project is termed "topological boundary wall". The specific context is related to a design program dealing with site conditions, programmatic aspects and constraints which vary along the length of a boundary line. The design material in this project attempts to apply topological conditions that maintain the same relations along the boarder line. It accommodates the new complexity of a certain topology, departing from the more static and typologically deterministic logic and design methodologies of the previous generation (see figure 1). The changing requirements found along the boundary create a constantly changing condition of context and program along the otherwise continuous design of the boundary. Together, the performance-based technique and the definition of parameters produce differentiation and heterogeneity in the design rather than the instantiation of a particular style, or standardized, modular structure as is currently routinely applied irrespective of complex changes of program and conditions.

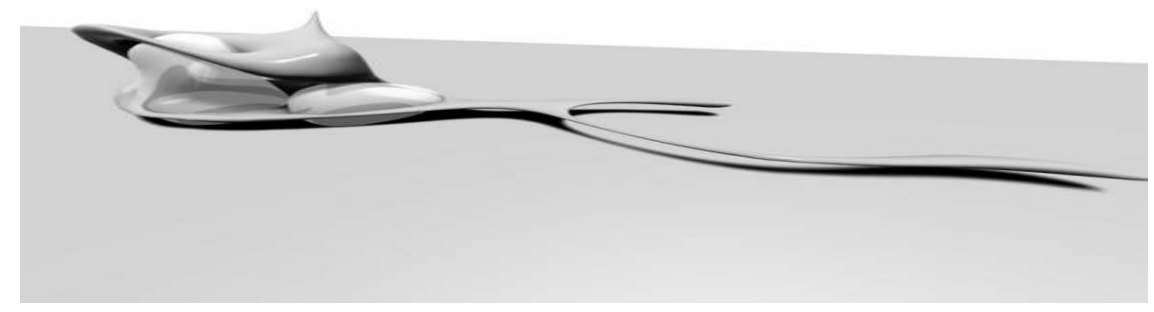

Figure 1: Topological design "The Dynamic Boundary" (designed by Farah Farah). 


\subsection{Parametric design "The Inner Space"}

The next project is termed a "parametric Inner Space". The digital material is defined as a structural and morphological system of parametric and responsive modules (see figure 2). The design process resulted in the production of parametric differentiation of the continuous material morphology responsive to light. Light conditions were selected as a context to test the applicability of this system. Different interpretations of small and large scale applications of the material systems were integrated as local and global scale of particularization. The context of the lighting demonstrated the applicability of this parametric approach to specific light conditions.

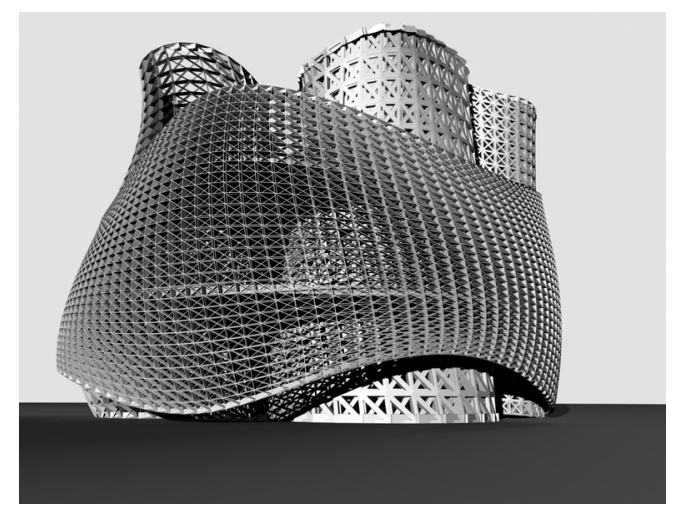

Figure 2: $\quad$ Parametric design (designed by Shoham Ben-Ari).

\subsection{Generative design: "The 'texlight' mechanism"}

The third project was termed "texlight mechanism". The conceptualization of the digital material is based on morphological principles of woven textiles. This woven material created an indeterminate range of heterogeneous folded profiles that were versions of folding and weaving principles. These profiles evolved to enable spatial, structural and environmental envelope functions within the woven matrix (see figure 3). The design transformations are defined by a set of syntactic rules (see figure 4). A Marina along the sea shore was selected as a context to inform the development of a continuously evolving structure.

\section{Summary and conclusions}

Our research has demonstrated that a new world view develops conceptual structures for design that may contradict the prevailing logic of design thinking. Rather than the employment of digital technologies, it is these emerging conceptual structures that strongly influence the logic of architecture and its design methods. These conceptual changes become the content of new pedagogical methods of design education. The awareness of change and conflicts 
can stimulate the necessary theorization and conceptualization for new approaches to design didactics. The "shock of the new" is not simply in the discovery of new formal vocabularies, but in the establishment of new approaches to design media. Among these, the election of the digital material as a suitable material morphology for a particular class of form generation has proved to be a productive and generative medium. Design thinking precedes design learning. The evolution of design thinking in the last decade now appears to have generated a new paradigm for design. As this paradigm crystallizes we first encounter it as a filed of conceptual conflicts between the prevailing and the new values of two design ontologies. New pedagogies can operate within this condition of the evolution and instability of ontologies. However, it can do so only by directly articulating and working with conceptual structures as pedagogical material. It is in this endeavor that we have established our studio for experimental didactics.

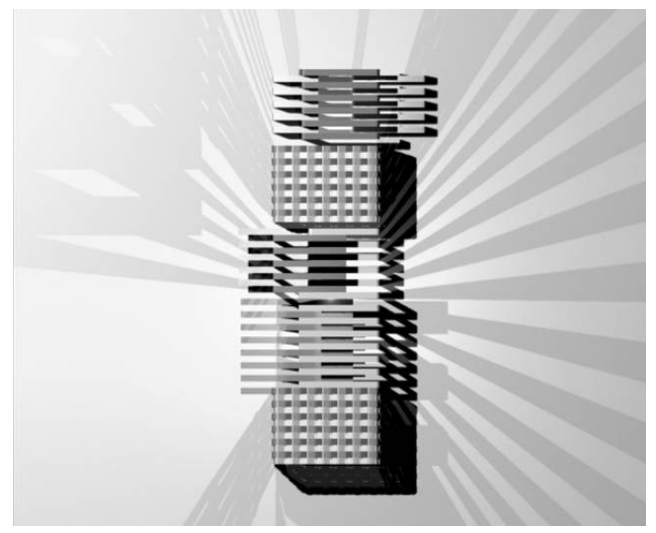

Figure 3: “Texlight mechanism” (designed by Alex Eitan and Tal Kasten).
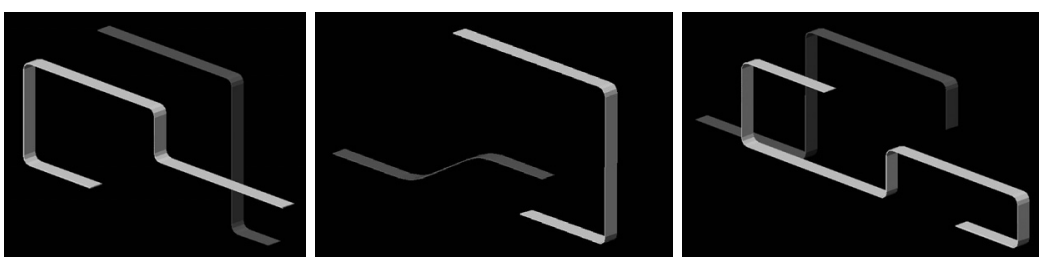

Figure 4: Generative-set (designed by Alex Eitan and Tal Kasten).

\section{Acknowledgements}

The following students are deeply acknowledged for their deep involvement and their creative work: Alex Eitan and Tal Kasten, Shoham Ben-Ari and Roey Hammer and Farah Farah. 


\section{References}

[1] Burry, M. Paramorph: Anti-accident Methodologies, in S. Perella. C (ed.) Hypersurface architecture II, Wiley, 1999

[2] Cross, N. (Ed) Developments in Design Methodology, John Wiley \& Sons, Chichester, UK, 1984

[3] Cross, N. Engineering Design Methods Strategies for Product Design (third edition), John Wiley and Sons Ltd., Chichester, UK, 2000

[4] Frazer, J. H. Creative Design and the Generative Evolutionary Paradigm, Bentley and Come, pp. 253-274, 2002

[5] Kolarevic B. Digital Morphogenesis, in Architecture in the Digital Age, in B. Kolarevic, (ed.): Architecture in the Digital Age, Spon Press, New York, 2003

[6] Kvan T. Mark, E. Oxman R. and Martens, B. Ditching the Dinosaur: Redefining the Role of Digital Media in Education, in International Journal of Design Computing, No.5, MIT Press, Cambridge, 2004

[7] Knight, T. and Stiny, G. Classical and Nonclassical Computation, Architectural Research Quarterly, 5 (4) pp. 355-372, 2001

[8] Lawson B. How Designers Think, Architectural Press, $3^{\text {rd }}$ Edition, London, 1997

[9] Liu, Y. T. $5^{\text {th }}$ FEIDAD Award: Demonstrating Digital Architecture, Birkhäuser, Berne, 2005

[10] Lindsey, B. Digital Gehry, Birkhäuser, Berne, 2002

[11] Lynn, G. Architectural Curvilinearity: the Folded, the Pliant and the Supple, in Lynn, G (ed.) Folding in Architecture, AD, Profile no. 102, pp. 8-15, 1993

[12] Lynn G. Animate Form Princeton Architectural Press, New York, 1999

[13] Oxman, R and Liu T (eds.) Cognitive and Computational Models in Digital Design. A Workshop of DCC04, First International Conference on cognition and computation in Design, MIT, Cambridge, U.S.A, 2004

[14] Oxman, R. The Conceptual Content of Digital Architecture - Content Analysis in Design, Electronic Journal of Arquiteturarevista, 1 (1), Unisinos, Brazil, 2005

[15] Oxman, R. Theory and Design in the First Digital Age, in R. Oxman (ed), a special issue on Digital Design, Design Studies, Elsevier, Vol 27 No 3., 2006

[16] Schön, D. The Reflective Practitioner. New York: Basic Books, 1983

[17] Schön, D. A and Wigging G. Kinds of Seeing and their Functions in Designing, in Design Studies 13 (2), pp. 135-15, 1988

[18] Stiny, G. (1980) "Introduction to shape and shape grammars," Environment and Planning B, pp. 349- 351

[19] Oxman Rivka (2003) Think-maps: teaching design thinking in design education, Design Studies, Volume 25, Number 1, pp. 63-91 